\title{
The First Determination of the Plasmid-Mediated Quinolone Resistance Determinants qnrA and qnrB from the Sudan
}

\author{
I.A. Malik ${ }^{1} *$ and K.M. Elhag ${ }^{2}$ \\ ${ }^{1}$ Liverpool Tropical school of Medicine.UK, PhD Medical Microbiology \\ (Liverpool University.UK), Ahfad University for Women-Sudan \\ ${ }^{2}$ Ahfad University for Women, Soba University Hospital-Sudan \\ *Corresponding author
}

\section{Keywords \\ qnr, plasmid, Genes, Sudan, Ciprofloxacin \\ Article Info \\ Accepted: \\ 12 February 2019 \\ Available Online: \\ 10 March 2019}

\begin{abstract}
A B S T R A C T
The aim of this study was to determine the prevalence of plasmid-mediated quinolone resistance $q n r$ genes (A, B and S) among a collection of enterobacterial clinical pathogens resistant to ciprofloxacin and was ESBL producers from Sudan. Seventy-two isolates that were ciprofloxacin resistant and ESBL producer were screened by PCR for $q n r$ genes (A, $\mathrm{B}$ and S). qnrA positive isolates were tested by PCR for class-1 integrons as well as resistance transfer by conjugation with sodium azide-resistant Escherichia coli J53. DNArelatedness was tested by PFGE. Qnr genes were detected among $13 \%$ of the test isolates, seven isolates were qnrB1 positive, included an E. coli and six Klebsiella pneumoniae isolates. qnrA was detected among two Enterobacter cloacae isolates. Genomic analysis by PFGE on the two qnrA positive isolates revealed two closely related organisms. Plasmid transfer of quinolone resistance was achieved on the two qnrA positive isolates, both plasmids showed part of the 3'-CS with the aminoglycoside-3'-adenyltransferases aadA2 and $\operatorname{aacA} 4$ genes as well as part of the Intl1 gene which was In6-like class-1 integrons. This study demonstrated high prevalence of $q n r \mathrm{~B}(10 \%)$ among the test isolates compared to $q n r \mathrm{~A}(3 \%)$ and non of the $q n r \mathrm{~S}$. This is the first report on plasmid-mediated ciprofloxacin resistance genes (qnrgenes) from Sudan.
\end{abstract}

\section{Introduction}

Quniolones are semi-synthetic antimicrobial agents developed in the 1960s. Fluoroquinolnes are newer synthetic generations with modified antimicrobial activity and broader spectrum of action. They have unsurpassed activity particularly against gram-negative bacteria (Strahilevitz et al.,
2009). They act by inhibiting bacterial isomerases enzymes, namely DNA gyrase and topoisomerases IV (Fluit et al., 2001). They are frequently used for the treatment of a variety of infections.

Fluoroquinolone resistance at high levels has been associated with multiple mutations in the chromosomal genes for the target enzymes 
and/or changes in expression of efflux pumps and porins (Martinez et al., 1998). However a plasmid-encoded mechanism of resistance of low level, has been described in 1998 (Martinez et al., 1998). Paterson and coworkers reported association between quinolone resistance and production of extended-spectrum $\quad \beta$-lactamase (ESBL) (Paterson et al., 2000).

The gene responsible for this resistance, $q n r$, is distinct from previously identified quinolone resistance genes (Tran and Jacoby, 2002). Quinlone-resistance genes, carried by a variety of gram-negative bacteria have been reported from different parts of the world (Wang et al., 2003; Jonas et al., 2005; Corkill et al., 2005; Nazic et al., 2005; Mammeri et al., 2005).

Association of $q n r$ gene with ESBL production among enterobacteriaceae has been reported by several workers (Mammeri et al., 2005 and Wang et al., 2003). And the ESBL producing enterobacteriaceae have been reported from different hospitals in the Sudan (Mekki et al., 2010) with high prevalence of blaCTX-M gene (unpublished data Malik and Elhag). Therefore I have taken up this study in order to find out the prevalence of plasmidmediated $q n r$ genes (A, B and S) among clinical isolates of ESBL producing enterobacteriaceae from the Sudan.

\section{Materials and Methods}

\section{Bacterial isolates}

Three hundred twenty four multidrug resistant bacterial isolates from wound and urine samples from patients who presented to the microbiology laboratories in Khartoum and Omdorman hospitals in the Sudan were studied. The strains were identified by standard microbiology methods (Forbes et al., 2007) and were stored at $-20^{\circ} \mathrm{C}$ in cryopreservers until tested.

\section{Susceptibility testing}

All 324 strains were tested for antibiotic susceptibilities by disc diffusion according to BSAC standardised methods. (Andrews.J.M, 2004), using Iso-Sensitest agar (Oxoid Ltd, Basingstoke, UK). Minimum inhibitory concentration (MIC) to nalidixic acid and ciprofloxacin were determined by the Etest method (AB Biodisk, Solna, Sweden).

\section{ESBL production}

Screening for ESBL production was determined by susceptibility to cefpodoxime and was confirmed phenotypically by the combined disc method (Carter et al., 2000). It was characterized genotypically by the detection of bla genes by PCR and nucleotide sequencing.

Strains of Enterobacteriaceae that were found to be quinolone-resistant and ESBLs producers were further screened by PCR for the presence of the qnr genes.

\section{Plasmid analysis and conjugation studies}

Conjugal transfer of resistance determinants was performed on broth culture with Escherichia coli J53 as recipient. After 24 hours of incubation at $37^{\circ} \mathrm{C}$, mating mixtures were platted onto agar supplemented with sodium azide $(100 \mathrm{mg} / \mathrm{L})$ and cefotaxime $(1 \mathrm{mg} / \mathrm{L})$. Plasmid DNA was prepared from donors and transconjugants using a commercial kit (Plasmid Mini Kit, Qiagen $\mathrm{GmbH}$, Hilden, Germany).

\section{Genomic analysis}

Restriction fragment length polymorphism (RFLP) was performed using restriction enzymes HindIII and EcoRI (Roche Diagnsotics Corporation, Indianapolis, USA). Pulsed field gel electrophoresis (PFGE) was performed with a CHEF DR III system (Bio- 
Rad, Hemel Hempstead, UK). DNA insert blocks were digested overnight with $\mathrm{Xba \textrm {I }}$ at $37^{\circ} \mathrm{C}$.

\section{PCR and nucleotide sequencing}

The DNA was extracted by suspending bacteria in a 5\% chelex 100 Resin slurry (BioRad Laboratories) in a sterile distilled water and then was boiled for 10 minutes. PCR was performed to detect the presence of $q n r$ genes (A and B) and class 1 integron cassette structures 3'-CS and 5'-CS conserved segments (integron variable region containing gene cassettes), intll integrase gene and sull conferring resistance to sulphonamides (Corkill et al., 2005). PCR products were detected by electrophoresis on $1 \%$ (w/v) agarose gels. Nucleotide sequence analysis was performed using the respective PCR primers for both directions using ABI 3100 automated sequencer (Warrington, UK), nucleotide sequence structures were compared with the data base using GenBank accession numbers: AY070235.

\section{Results and Discussion}

Seventy two isolates were ESBL producers and resistant to quinolones. Nine of these isolates $(13 \%)$ were positive for the qnr genes. They included one E. coli, six Klebsiella pneumoniae and two Enterobacter cloacae (table 1).

Sequence analysis of the $q n r$ amplicons revealed that seven $(10 \%)$ of the test isolates were $q n r \mathrm{~B} 1$ positive (Fig. 1) and the remaining two (3\%) were qnrA1 positive (Fig. 2). $q n r \mathrm{~S}$ was not detected among these isolates.

All the $q n r$ positive isolates were positive phenotypically and by PCR for extendedspectrum $\beta$-lactamases (ESBL) production, and expressed blaSHV and/or blaCTX-M. They were negative for Amp-C production.
qnrA positive isolates were subjected to conjugation and plasmid transfer experiments as well as integron class- 1 studies. Quinolone resistance was detected on both E. coli J53 transconjugants (TCJs) crossed with $E$. cloacae 1 and 2 and was transferred on a plasmid with the size of $96.9 \mathrm{Kbp}$ (Fig. 3).

Conjugation-experiments were performed on both isolates and each transferred the resistance to nalidixic acid and ciprofloxacin. Other groups of antibiotics were also tested (table 2). Results showed transfer of resistance to amoxicillin, cefpodoxime, gentamicin, trimethoprim, nalidixic-acid, aztreonam, ceftazidime and ciprofloxacin to the recipient organism E.coli J53.

The Minimum inhibitory concentration (MIC) for both Nalidixic acid and ciprofloxacin was determined on the two tranconjugant organisms as well as the recipient organism (table 3).

Plasmid extraction was performed on both donor and transconjugant organisms. A plasmid of $96.9 \mathrm{kbp}$ in size was responsible for resistance transfer from the donor to the recipient organisms for both isolates (Fig. 3).

Nalidixic acid resistance on E.coli-TCJ-1 was increased by 8 folds (MIC $3.0-24 \mathrm{mg} / \mathrm{L}$ ) and for E.coli-TCJ-2 increased by 11 folds (MIC 3.0-32mg/L). Resistance to ciprofloxacin for both isolates increased by 16 folds (MIC 0.006-0.094mg/L).

RFLP studies revealed two heterogeneous genotypic patterns. Genomic analysis by PFGE on the two qnrA positive isolates revealed two closely related organisms. Integron studies showed part of the 3'-CS with the aminoglycoside-3'-adenyltransferases $\operatorname{aad} \mathrm{A} 2$ and $\operatorname{aac} \mathrm{A} 4$ genes as well as part of the Intl1 gene which was In6-like class-1 integrons. 
Table.1 qnr and ESBL positive enterobacterial isolates detected within the clinical pathogens from the Sudan

\begin{tabular}{|l|l|l|l|l|l|}
\hline $\begin{array}{l}\text { Isolate } \\
\text { No. }\end{array}$ & $\begin{array}{l}\text { Bacterial } \\
\text { isolate }\end{array}$ & $\begin{array}{l}\text { Source } \\
\text { specimen }\end{array}$ & $\begin{array}{l}\text { of } \\
\text { ESBL- } \\
\text { phenotype }\end{array}$ & blagene & $\begin{array}{l}\text { qnr- } \\
\text { gene }\end{array}$ \\
\hline QNR1 & E.cloacae-1 & Urine & + & SHV5a & qnr-A1 \\
\hline QNR2 & E.cloacae-2 & Urine & + & CTX-M, SHV5 & qnr-A1 \\
\hline QNR3 & E.coli & Urine & + & SHV5 & qnr-B1 \\
\hline QNR4 & K.pneumoniae & Wound & + & $\begin{array}{l}\text { CTX-M, } \\
\text { SHV11 }\end{array}$ & qnr-B1 \\
\hline QNR5 & K.pneumoniae & Urine & + & $\begin{array}{l}\text { CTX-M, } \\
\text { SHV5a }\end{array}$ & qnr-B1 \\
\hline QNR6 & K.pneumoniae & Urine & + & $\begin{array}{l}\text { CTX-M, } \\
\text { SHV5a }\end{array}$ & qnr-B1 \\
\hline QNR7 & K.pneumoniae & Urine & + & CTX-M, SHV5 & qnr-B1 \\
\hline QNR8 & K.pneumoniae & Wound & + & $\begin{array}{l}\text { CTX-M, } \\
\text { SHV11 }\end{array}$ & qnr-B1 \\
\hline QNR9 & K.pneumoniae & Urine & + & CTX-M & qnr-B1 \\
\hline
\end{tabular}

Table.2 Disc-diffusion results for the qnr-A transconjugant organisms, donors and the recipient organism $(\mathrm{J} 53)$. (T.C $=$ Transconjugant organism, N.R= not recorded $)$

\begin{tabular}{|l|l|l|l|l|l|l|}
\hline & \multicolumn{7}{|c|}{ Zone Size (mm) } \\
\hline Antibiotic & Recipient & Donor-1 & T.C-1 & Donor-2 & T.C-2 & Breakpoints(R $\leq$ ) \\
\hline Ampicillin & 23 & 0 & $<6$ & 0 & $<6$ & 11 \\
\hline Augmentin & 28 & 12 & 20 & 11 & 18 & 11 \\
\hline Cefpodoxime & 33 & 10 & 14 & 16 & 16 & 25 \\
\hline Gentamicin & 25 & 8 & 8 & 11 & 11 & 16 \\
\hline Ciprofloxacin & 34 & 0 & 27 & 0 & 28 & 16 \\
\hline Trimethoprim & 26 & 0 & $<6$ & 0 & $<6$ & 14 \\
\hline Nalidixic-acid & 28 & 0 & 16 & 0 & 15 & 17 \\
\hline Meropenem & 38 & 30 & 39 & 32 & 40 & 22 \\
\hline Amikacin & 27 & 18 & 27 & 19 & 25 & 15 \\
\hline Aztreonam & 42 & N.R & 21 & N.R & 24 & 23 \\
\hline Tazobactam & 36 & N.R & 28 & N.R & 30 & 22 \\
\hline Ceftazidime & 38 & 10 & 22 & 14 & 22 & 27 \\
\hline & & & & & & \\
\hline
\end{tabular}

Table.3 MIC results for both qnr-A transconjugants and recipient organism (J53)

\begin{tabular}{|l|l|l|l|}
\hline \multicolumn{2}{|c|}{ Organism } & MIC $(\boldsymbol{\mu g} / \mathrm{L})$ \\
\hline E.coli & J53 & 3 & \\
\hline Entero.cloacae & T.C 1 & 24 & 0.006 \\
\hline Entero.cloacae & T.C 2 & 32 & 0.094 \\
\hline
\end{tabular}


Fig.1 PCR qnr-B positive isolates (E.coli and Klebsiella spp).

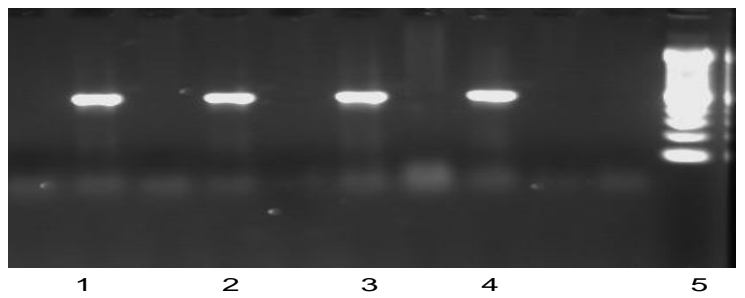

Lane 1: qnrB positive E.coli isolate. Lane 2, 3: qnrB positive Klebsiella isolates. Lane 4: positive control. Lane 5: Lambda DNA of a multiple 100 bp size marker

Fig.2 PCR qnr-A positive isolates.

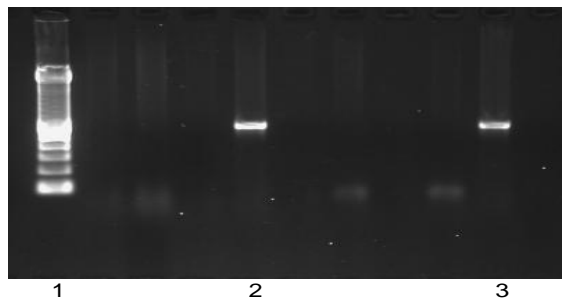

Lane 1: Lambda DNA of a multiple $100 \mathrm{bp}$ size marker. Lane 2 and 3: qnr-A positive isolates.

Fig.3 Plasmid extraction of the qnrA donor and transconjugants

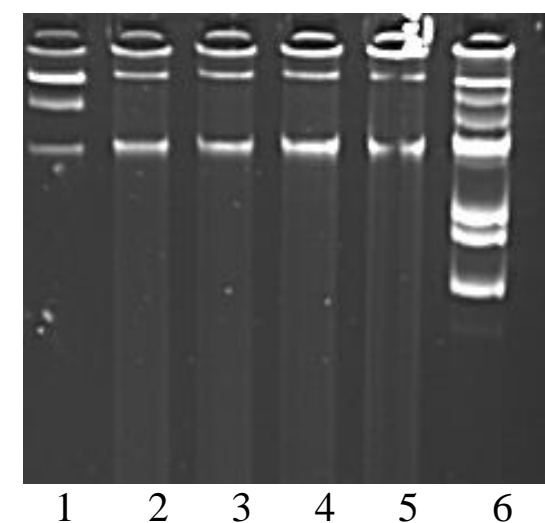

Lane 2, 3, 4 and 5: plasmid of the size $96.9 \mathrm{Kbp}$ in $q n r-A$ positive isolates. Lane 1 and 6: the markers 39R and V517 respectively. 
Fluoroquinolones have been introduced with a broader spectrum of activity, to rival betalactams and macrolides in clinical practice (Takahashi et al., 2003). However, in the Sudan quinolones are excessively used for the treatment of a wide variety of infections. Together with cephalosporins they remain the main injectable antimicrobials available for general clinical use. Thus antimicrobial resistance to quinolones and beta-lactams is rising (Ibrahim et al., 2012). No wonder, as these agents have been described as causative of collateral damage (Paterson et al., 2004).

In this study $q n r$ genes were detected among $13 \%$ of the isolates. qnr B genes constitute the majority ( $10 \%$ ) as reported by some studies (Jacoby et al., 2006; Tamanag et al., 2008; Leila et al., 2015).

All $q n r$ positive isolates from this study were ESBL producers expressing blaCTX-M and/or blaSHV genes (blaSHV5, blaSHV5a or blaSHV11).

In this study Quinolone resistance transfer was clearly demonstrated by the conjugation study performed on the qnrA positive isolates, and was explained by the rise in resistance levels of the transconjugate organisms by 8 11 folds and 16 folds to nalidixic acid and ciprofloxacin respectively. qnrA genes on the two test isolates from the present study were located on plasmids of molecular size $\sim 96 \mathrm{kbp}$ encoding varying resistance profiles.

Qnr and ESBL resistance determinants were co-transferred on the same plasmid with resistance determinants to other antimicrobials (gentamicin, trimethoprimsulphamethoxazole, chloramphenicol and tetracycline). This finding also confirms the co-association of plasmid mediated resistance determinants of ciprofloxacin and ESBL resistance determinants that has been reported by many other studies (Paterson et al., 2000;
Jonas et al., 2005; Corkill et al., 2005; Nazic et al., 2005; Mammeri et al., 2005).

Integron studies carried-on qnrA positive isolates had demonstrated the possibility of carriage of class-1 integron on these plasmid.

The majority of these isolates were uropathogenic isolates collected from two different hospital settings in the capital of the Sudan, where Quinolones are widely used in the treatment of Urinary tract infections. The findings from this study with other few previous studies from the Sudan that demonstrated the rising resistance prevalence to ciprofloxacin and other antimicrobials (Ibrahim et al., 2012), with the development of multidrug resistant pathogens harbouring different genes and mechanisms of resistance to the most essential and lifesaving antimicrobials, clearly reflect the critical situation of the future of antimicrobials in such a developing country.

\section{Acknowledgments}

A special thanks to Mr John. E. Corkill and to Professor Ahmed Abdel Mageed for their supervision and help. This work is dedicated to the soul of late Professor C.A. Hart.

\section{References}

Andrews J M. 2004. BSAC standardized disc susceptibility testing method (version 3 ). $J$ Antimicrob Chemother. 53: 713-28.

Carter MW, Oakton KJ, Warner M and Livermore DM. 2000. Detection of ExtendedSpectrum beta-lactamases in Klebsiellae with the Oxoid combination disk method. J Clin Microbiol.38:4228-4232

Corkill JE, Anson JJ and Hart CA. 2005. High prevalence of the plasmid-mediated quinolone resistance determinant qnrA in multidrug-resistant Enterobacteriaceae from blood cultures in Liverpool, UK. Journal of Antimicrobial Chemotherapy. 56: $1115-1117$ 
Fluit AC, Visser MR and Schmitz FJ. 2001.Molecular detection of antimicrobial resistance Clin Microbiol Rev.14:836-71

Forbes BA, Sahm DF and Weissfield AS. 2007. Overview of bacterial identificationMethods and strategies. Baily and Scott's Diagnostic Microbiology, $12^{\text {th }}$ edition. Mosby Elsevier).

Ibrahim ME, Bilal NE and Hamid ME. 2012. Increased multi-drug resistant Escherichia coli from hospitals in Khartoum state, Sudan. Afr Health Sci. 12:368-375

Jacoby GA, Walsh KE, Mills DM et al. 2006. $q n r B$ another plasmid-mediated gene for quinolone resistance. Antimicrob. Agents Chemother. 50:1178-1182.

Jonas D, Biehler K, Hartung D et al. 2005. Plasmid-mediated quinolone resistance in isolates obtained in German Intensive Care Units. Antimicrobial agents Chemotherapy. 49: 773-5.

Leila V, Ali AD, Mehrez MJ. et al. 2015.The emergence of plasmid mediated quinolone resistance qnrA2 in extended spectrum $\beta$ lactamase producing Klebsiella pneumoniae in the Middle East. Daru . 23: 34

Martinez-Martinez L, Pascual A and Jacoby GA. 1998. Quinolone resistance from a transferable plasmid. Lancet. 351: 797-9

Mammeri H, Van De Loo M, Poirel L et al. 2005. Emergence of plasmid mediated quinolone resistance in Escherichia coli in Europe. Antimicrob Agents Chemother . 49: 71-6

Mekki AH, Hassan AN, Elsayed DM. 2010. Extended spectrum beta lactamases among multidrug resistant Escherichia coli and Klebsiella species causing urinary tract infections in Khartoum. Journal of Bacteriology research. 11(3):18-21.

Nazic H, Poirel L, Nordmann P. 2005.Further identification of plasmid mediated quinolone resistant determinant in Enterobacteriaceae in Turkey. Antimicrob Agents Chemother. 49: 2146-7.

Paterson DL, Mulazimoglu L, Casellas JM et al. 2000. Epidemiology of ciprofloxacin resistance and its relationship to extendedspectrum beta-lactamase production in Klebsiella pneumoniae isolates causing bacteraemia. Clin Infect Dis. 30: 473-8

Paterson DL. 2004 "Collateral Damage" from cephalosporins or quinolone antibiotic therapy. Clin Infect Dis.38 (supp 4):S3415

Strahilevitz J, Jacoby GA, Hooper DC. et al. 2009. Plasmid-mediated quinolone resistance: a multifaceted threat. Clin Microbiol Rev.22:664-89.

Takahashi H, Hayakawa I, Akimoto T. 2003.The history of the development and changes of quinolone antibacterial agents. Yakushigaku Zasshi.38:161-79

Tamang MD, Seol SY, Oh JY. et al. 2008. Plasmid-mdiated quinolone resistance determinants $q n r A$, qnrB, and $q n r S$ among clinical isolates of Enterobacteriaceae in a Korean hospital. Antimicrob. Agents Chemother. 52:4159-4162

Tran JH and Jacoby GA. 2002. Mechanism of plasmid- mediated quinolone resistance. Proc Natl Acad Sci USA.99:5638-42

Wang M, Tran JH, Jacoby GA et al. 2003. Plasmid-mediated quinolone resistance in clinical isolates of Escherichia coli from Shanghai, China. Antimicrob Agents Chemother. 47: 2242-8.

Wang M, Sahm DF, Jacoby GA. 2004. Emerging plasmid mediated quinolone resistance associated with qnr gene in Klebsiella pneumoniae clinical isolates in the United States. Antimicrob Agents Chemother. 48:1295-9

\section{How to cite this article:}

Malik, I.A. and Elhag, K.M. 2019. The First Determination of the Plasmid-Mediated Quinolone Resistance Determinants qnrA and qnrB from the Sudan. Int.J.Curr.Microbiol.App.Sci. 8(03): 1612-1618. doi: https://doi.org/10.20546/ijcmas.2019.803.187 